\title{
ROBOT DA VINCI: EL QUIRÓFANO DEL FUTURO
}

\section{DA VINCI ROBOT: THE OPERATING ROOM OF THE FUTURE}

\author{
Ricardo John Palomares Orihuela ${ }^{1}$
}

\section{RESUMEN}

Por siglos, el hombre ha combatido de diferentes maneras las enfermedades que atacan el organismo humano. La evolución tecnológica aplicada en medicina se ha dado desde la aparición de los primeros galenos, quienes usaron rústicas herramientas de piedra y metal, hasta los de hoy en día, quienes hacen uso de los progresos tecnológicos a través de herramientas innovadoras y efectivas para salvar vidas humanas $y$ erradicar las enfermedades con mayor efectividad.

El desarrollo de la robótica, ampliamente utilizado en el proceso industrial, ha permitido una mayor optimización de recursos y una mayor precisión. Y este no ha quedado ajeno a la medicina. Es así como hoy en día se utiliza el robot Da Vinci exitosamente en cirugías cardiacas. La cirugía robótica representa, a nivel mundial, el último avance tecnológico en procedimientos quirúrgicos mínimamente invasivos.

Palabras clave: robots, medicina, cirugía robótica, centro quirúrgico

\begin{abstract}
For centuries, man has fought in different ways diseases that attack the human body. Technological developments applied in medicine, have been since the advent of the first doctors who used stone tools and rustic metal, to today, who make use of technological advances through innovative and effective tools to save human lives and eradicate diseases more effectively. The development of robotics, widely used in the industrial process, has enabled further optimization of resources and greater accuracy, it has not been apart from medicine. Thus, today the Da Vinci robot is used successfully in cardiac surgery. Robotic surgery represents worldwide the latest technological advancement in minimally invasive surgical procedures.
\end{abstract}

Keywords: robots, medicine robotic surgery, surgical center

1 Magíster en Ciencias de la Educación con mención en Docencia Universitaria, Maestría en Ciencias de la Electrónica con mención en Ingeniería Biomédica, Ingeniero Mecatrónico, Docente de la Escuela Profesional de Ingeniería Mecatrónica, Facultad de Ingeniería, Universidad Ricardo Palma. <rpalomares@ieee.org> 


\section{INTRODUCCIÓN}

Desde los inicios de la civilización, el ser humano ha construido máquinas que imitan el funcionamiento de las partes móviles del cuerpo humano. Los antiguos egipcios unieron brazos mecánicos a estatuas que representaban a sus dioses, los cuales eran operados por sacerdotes, quienes clamaban que el movimiento era inspiración de los dioses.

El concepto tradicional de cirugía, que implica cortar, coser y tocar los órganos y tejidos del paciente, se vuelve obsoleto. La miniaturización de las herramientas quirúrgicas, los avances en el procesamiento de señales de vídeo, las imágenes en tres dimensiones y las técnicas endoscópicas facilitan las intervenciones quirúrgicas no invasivas, incluso en zonas del cuerpo humano de muy difícil acceso.

La primera cirugía con robots fue realizada en 1985, en el campo de la neurocirugía. Se trató del PUMA 560, que se utilizó para orientar una biopsia cerebral. En 1988, se utilizó el robot PROBOT en una resección prostática transuretral. [1]

En 1992, la International Business Machine (IBM) desarrolló el ROBODOC, utilizado en cirugía ortopédica. Este robot fue utilizado por primera vez en el reemplazo de caderas, y fue el primer robot aprobado por la Food and Drug Administration (FDA) en cirugía. Ese mismo año, la National Aeronautics and Space Administration (NASA) solicitó a la compañía Computer Motion la creación de un brazo robótico para las tareas espaciales. En 1994, la FDA aprobó el uso del Automated Endoscopic System for Optimal Positioning (AESOP), desarrollado por Computer Motion. Se trataba de un brazo mecánico para sostener la cámara laparoscópica, el cual se muestra en la Figura 1, lo que permite reemplazar al médico asistente en esta tarea. Los movimientos del brazo eran controlados manualmente o a distancia. Posteriormente, se adicionó al robot un sistema de control por voz. [1]

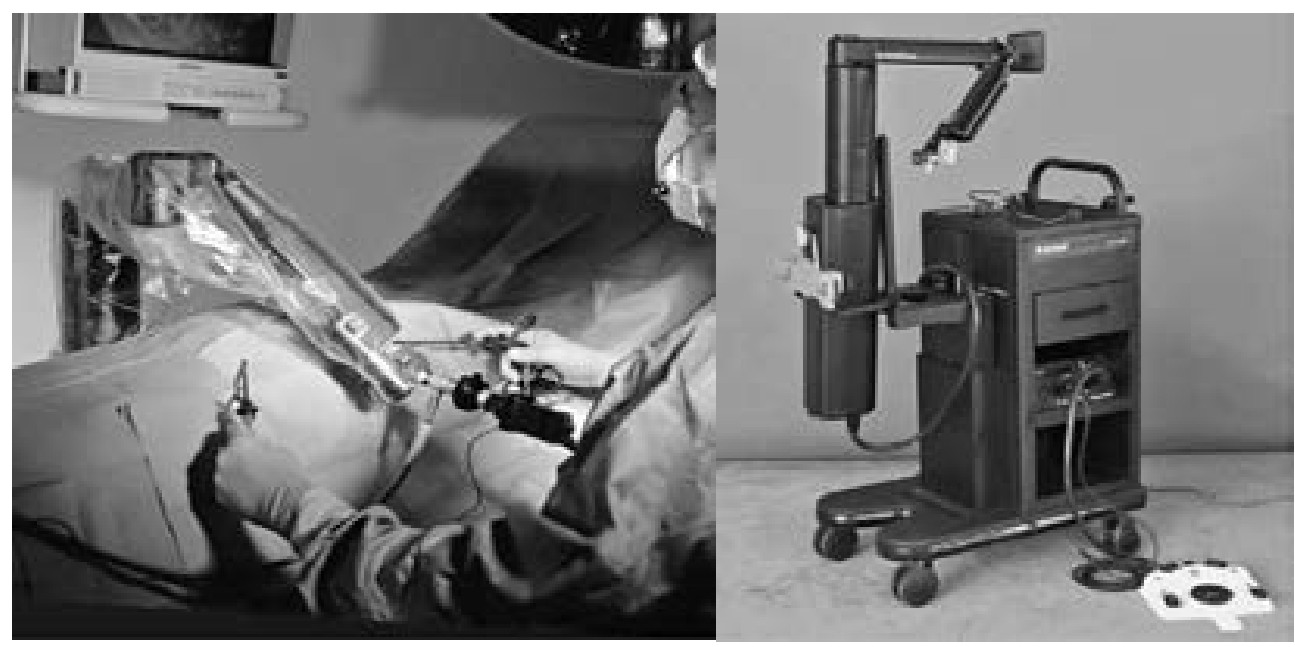

Figura 1: Robot AESOP (Automated Endoscopic System for Optimal Positioning) Fuente: http://twcroboticsurgery.weebly.com/past.html

Otro sistema para el manejo de una cámara fue el ENDOASSIST, creado por Amstrong Health. La diferencia con el sistema AESOP está en el control de los movimientos de la cámara a través de un sistema infrarrojo accionado por los movimientos de la cabeza del cirujano al observar el monitor que proyecta la imagen obtenida. 
El Departamento de Defensa de Estados Unidos, interesado en la posibilidad de realizar cirugías a distancia en posibles ambientes bélicos, impulsó el desarrollo de la telerrobótica bajo el proyecto denominado Mobile Advanced Surgical Hospital (MASH). Así, nace el Da Vinci Surgical System, creado por Integrated Surgical System. Con este mismo propósito, la compañía Computer Motion creó, en 1998, el sistema ZEUS (sistema de control digital para operar a distancia los instrumentos laparoscópicos). El primer sistema de visión en tres dimensiones para laparoscopia fue el ENDOWRIST de Integrated Surgical System.

Durante el año 2000, la FDA aprobó el uso de estos sistemas para EE. UU., pero, aun cuando se trata de un sistema que permite realizar cirugías a distancia, se aprobó utilizar los comandos de operación a distancia en la misma sala operatoria donde se encuentra el paciente. En junio de 2003, ambas compañías se fusionan bajo el nombre único de Intuitive Surgical Inc., y unen las tecnologías en el desarrollo del Sistema Integrado Da Vinci en el desarrollo la cirugía laparoscópica robótica.

\section{CIRUGÍA ROBÓTICA: EL ROBOT DA VINCI}

La cirugía robótica representa, a nivel mundial, el último avance tecnológico en procedimientos quirúrgicos mínimamente invasivos, y da como resultado el sistema quirúrgico Da Vinci, el cual está accionado por tecnología robótica de vanguardia. El sistema permite graduar, filtrar y transformar los movimientos de las manos del cirujano en movimientos precisos de los brazos robóticos que sostienen los microinstrumentos. [2]

El sistema Da Vinci aumenta la precisión quirúrgica y permite llevar a cabo cirugías complejas a través de pequeñas incisiones quirúrgicas. El sistema no se puede programar ni puede tomar decisiones por sí mismo, sino que requiere que cada maniobra quirúrgica se realice con la intervención directa del cirujano.

Los robots quirúrgicos han sido diseńados con la finalidad de que pueden asistir al cirujano en un procedimiento quirúrgico. Por ejemplo, en los procedimientos por laparoscopía, se introducen, por pequeños orificios en el abdomen, tres dispositivos: una cámara óptica y dos pinzas que pueden llevar desde piezas instrumentales como micropinzas hasta los bisturís, tal como se muestra en la Figura 2. [3]

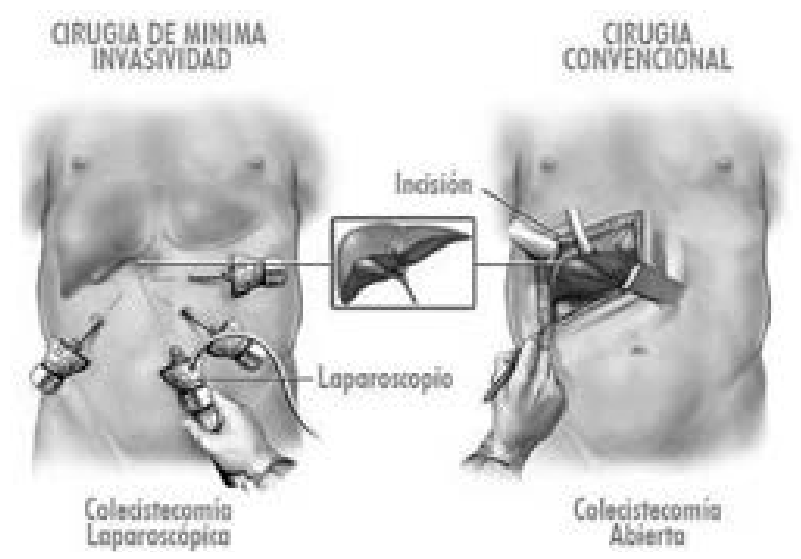

Figura 2: Cirugía minimamente invasiva vs. Cirugía convencional

Fuente: http://iqaquiron.com/portallindicaciones-de-la-colecistectomia-en-pacientes-de-cirugia-bariatrical 
El sistema Da Vinci combina la visión en tres dimensiones con instrumentos con un rango de movilidad superior, lo que entrega un grado de precisión mayor. Además, posee un sistema de control ergonométrico. Estas características permitirán una reducción del trauma causado por una cirugía, reducción de las pérdidas sanguíneas, menos dolor, recuperación más rápida, retorno a la vida laboral más rápido, mejores resultados cosméticos y una mayor precisión para realizar procedimientos de mayor complejidad. Todo esto a través de sus diferentes componentes, que se muestran en la Figura 3.
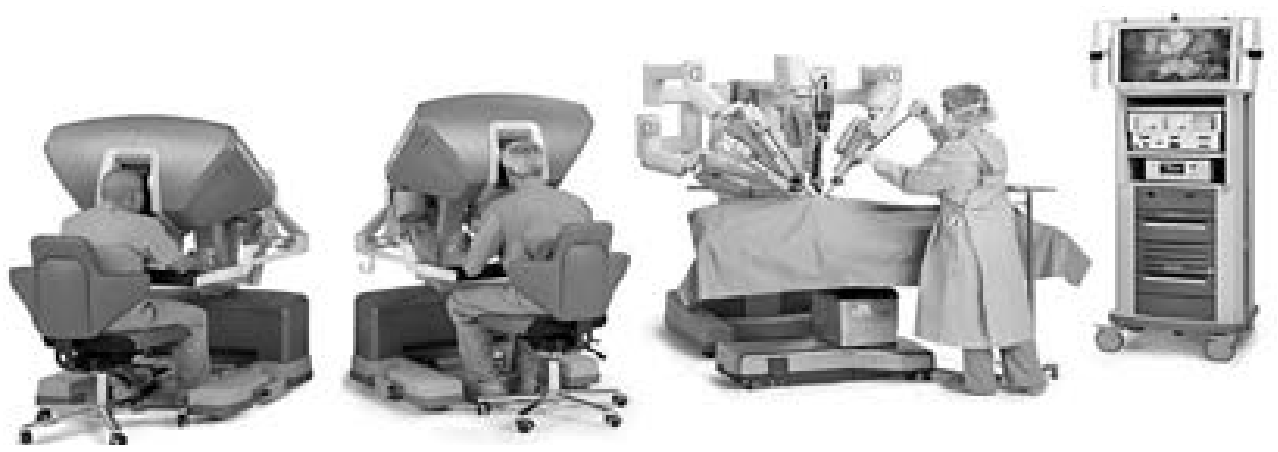

Figura 3: Sistema Da Vinci

Fuente: http://www.cirugiatoracicarobotica.es/

El soporte con brazos robóticos consiste en una armazón que se coloca al lado de la camilla del paciente, en el que se fijan cuatro brazos robóticos a través de los cuales se manejan a distancia los instrumentos y la cámara. Los dos primeros brazos representan la mano derecha y la izquierda respectivamente. El tercer brazo sostiene el endoscopio, que se controla desde la consola, por lo que no requiere otro cirujano para sostener la cámara. El cuarto brazo es opcional y puede ser utilizado en tareas adicionales, tal como se muestra en la Figura 4. [4]

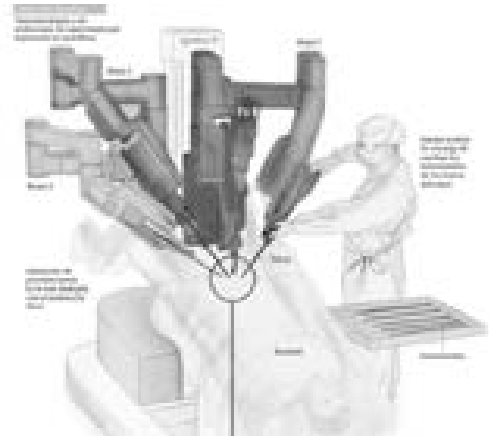

(a)

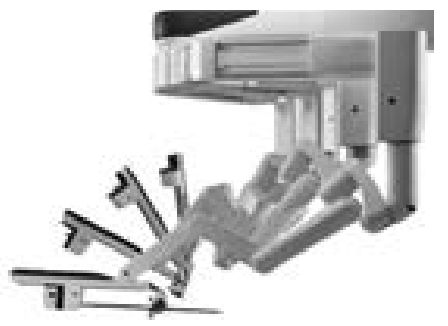

(b)

Figura 4: Soporte de brazos robóticos: (a) esquemático y (b) real

Fuente: http://www.cun.es/enfermedades-tratamientos/tratamientos/cirugia-robotica-da-vinci

La consola quirúrgica se trata de un sistema que permite controlar a distancia los brazos del robot. Permite que el cirujano se apoye, sentado, y aproveche las características ergonométricas para realizar la cirugía. Está compuesto por una zona de visión, un mecanismo de control para ambas manos y pedales para accionar los diferentes instrumentos, tal como se muestra en la Figura 5. [4] 


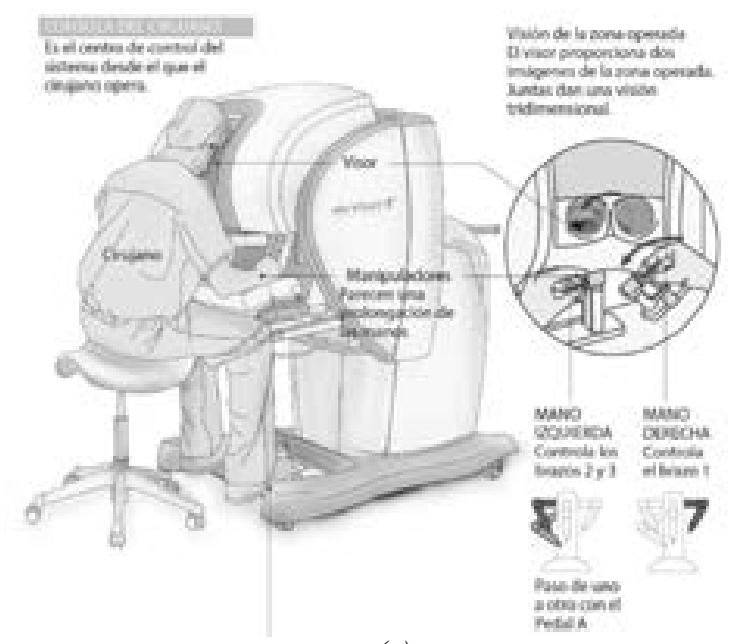

(a)

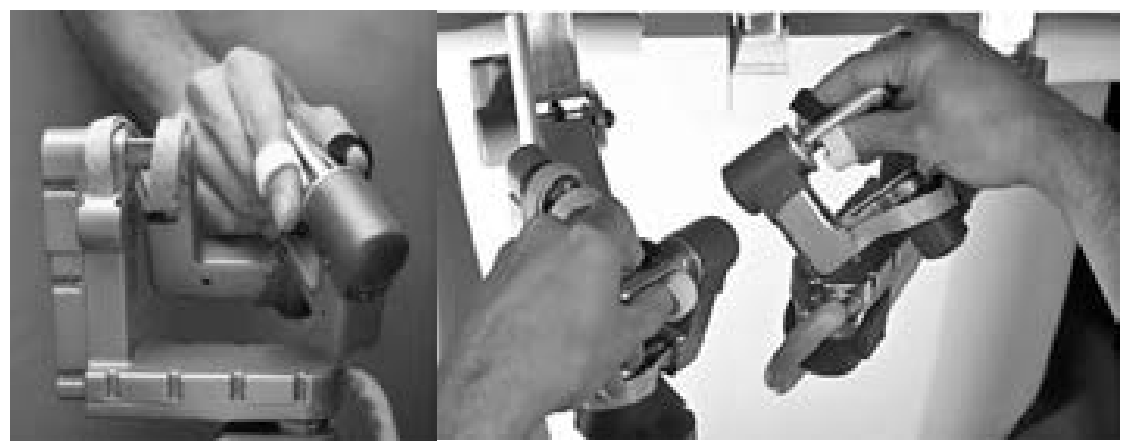

(b)

Figura 5: Consola quirúrgica y mandos ergonómicos: (a) esquemático y (b) real Fuente: http://www.cun.es/enfermedades-tratamientos/tratamientos/cirugia-robotica-da-vinci

El sistema de visión es un sistema de alta resolución que entrega una visión magnificada en tres dimensiones. Este sistema incorpora la posibilidad de acercamiento instantáneo controlado desde los comandos a distancia, lo que permite al cirujano sentirse inmerso en el campo operatorio, y proporciona una mayor precisión para la manipulación de tejidos. Esto se complementa con un sistema de iluminación de gran intensidad. El telescopio es de $12 \mathrm{~mm}$ y posee, en su interior, dos cámaras de $5 \mathrm{~mm}$ cada una. Estas imágenes generan un campo operatorio virtual que puede observarse con una visión binocular en la consola de telecomando, tal como se muestra en la Figura 6. [2]

El sistema Da Vinci posee cámaras de alta definición (HD) con lentes dobles de $30^{\circ}$ de angulación, tal como se muestra en la Figura 7.

Respecto a los instrumentos específicos para la cirugía, los efectores terminales con endowrist se han diseńado con 7 grados de movimiento, que imitan la destreza de la mano y muñeca humana, tal como se muestra en la Figura 8. Esto permite superar una de las desventajas de la laparoscopia tradicional, que posee un rango de movimiento limitado. Cada instrumento ha sido diseñado específicamente, lo que permite clampear, suturar o manipular los diferentes tejidos en los cuales se realiza la cirugía. [5] 


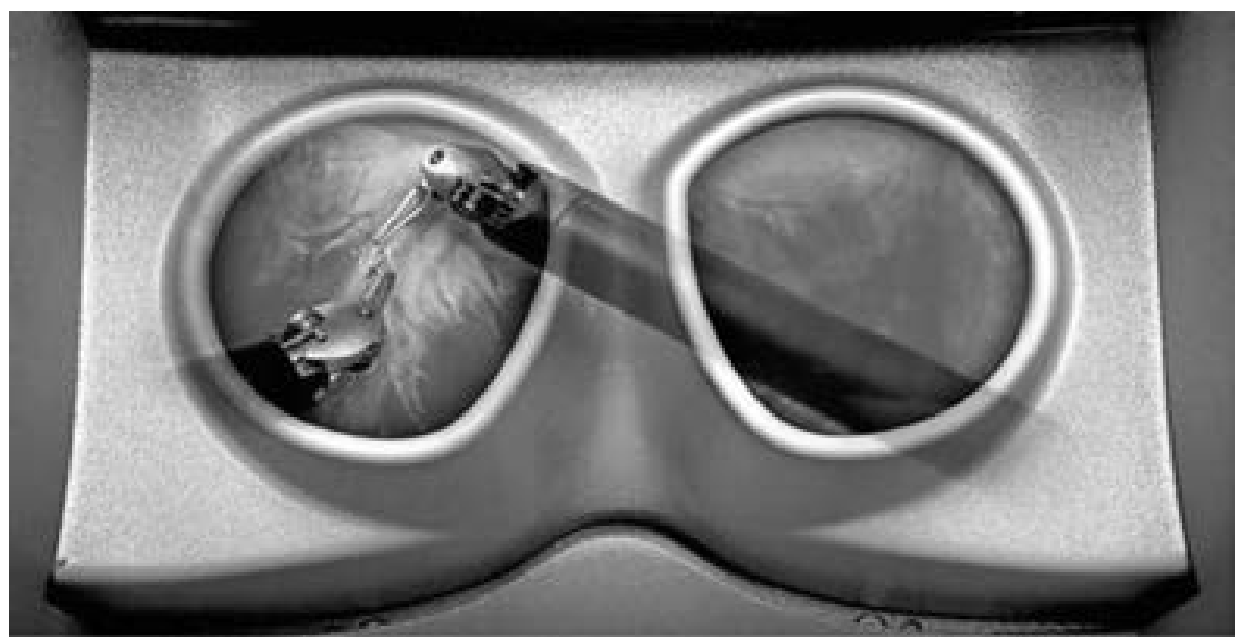

Figura 6: Visión binocular en la consola de telecomando

Fuente: http://www.australianroboticsurgery.com.aul

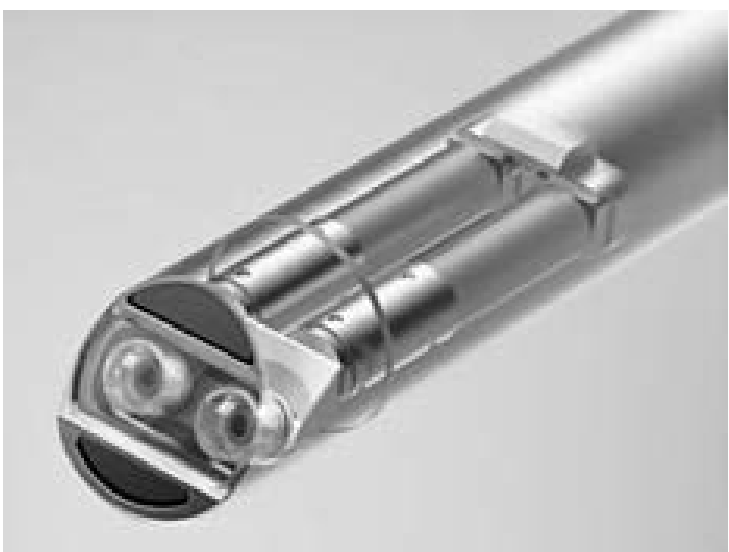

Figura 7: Cámara de alta definición HD

Fuente: http:/lallaboutroboticsurgery.com/september2010.html

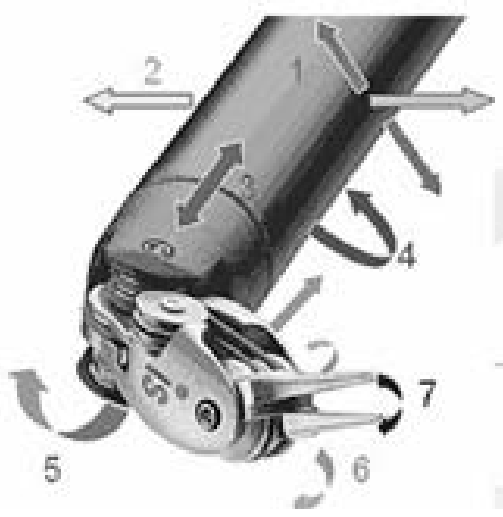

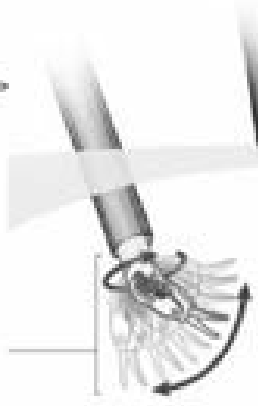

imitan los. movimientos de b mano.

(a)

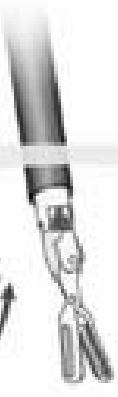

Endoscopio

Dos cámaras captan

La imagen en 30 . 


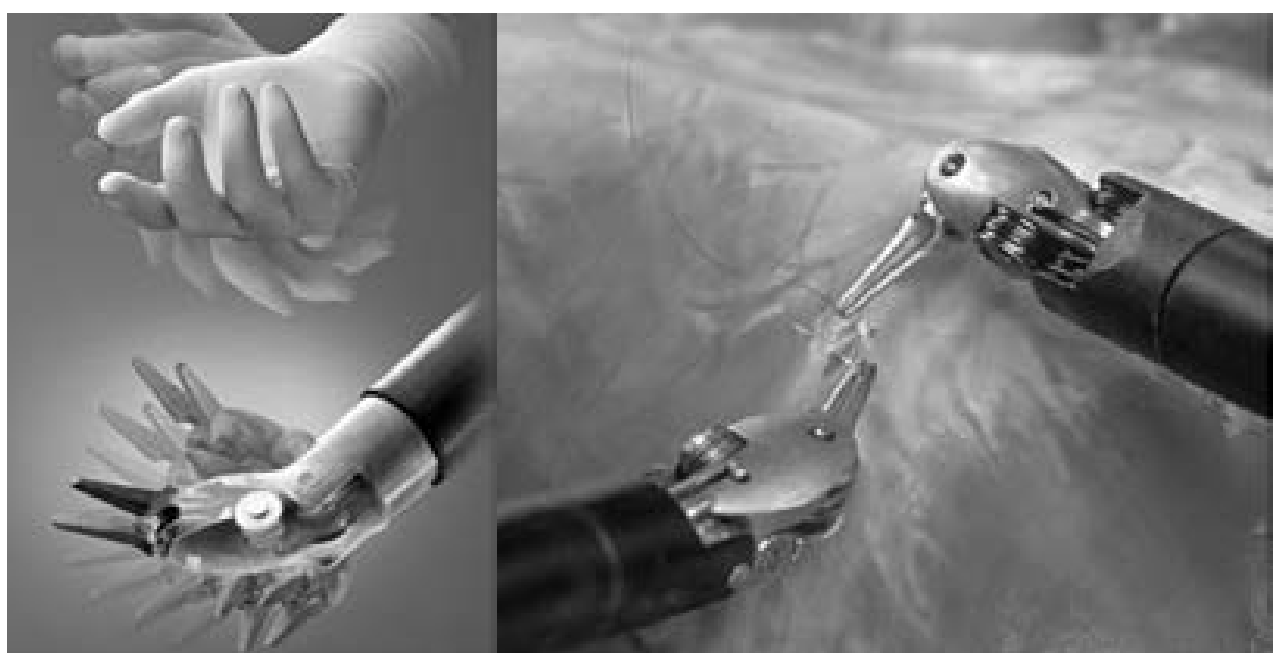

(b)

Figura 8: Efectores terminales endo-wrist: (a) esquemático y (b) real Fuente: http://www.intuitivesurgical.com/products/instruments/

Computer Motion e Intuitive Surgical recibieron la aprobación regulatoria en América del Norte, Europa y Asia para que sus robots sean utilizados en cirugías invasivas mínimas. En la actualidad, existen más de 2500 robots quirúrgicos Da Vinci en el mundo con aplicaciones en urología, ginecología, cirugía general, cirugía pediátrica, cirugía torácica y cirugía cardiaca. [6]

\section{CONCLUSIONES}

La medicina ha encontrado, en la ingeniería, a un aliado en el que ha depositado sus esperanzas para perfeccionar sus técnicas de soporte y asistencia al ser humano mediante el uso de robots que le permitan maximizar el rendimiento de sus procesos quirúrgicos. En el futuro, la robótica médica pretende compatibilizar al médico cirujano con un robot con la finalidad de mejorar los resultados en un procedimiento quirúrgico.

El sistema quirúrgico Da Vinci no puede programarse ni tampoco tomar decisiones en forma independiente. En lugar de eso, este sistema necesita una orden directa del cirujano para realizar cada maniobra quirúrgica.

El sistema Da Vinci se ha utilizado con éxito en decenas de miles de procedimientos mínimamente invasivos en todo el mundo. Ofrece múltiples características de seguridad redundantes que convierten al robot Da Vinci en el método más efectivo y menos invasivo, y potencialmente tan seguro como los métodos quirúrgicos tradicionales.

Asimismo, proporciona un rango de movimiento mayor que el de la mano humana, con movimientos más ergonómicos y precisos que con la laparoscopia convencional.

El sistema quirúrgico Da Vinci evita los temblores y controla los movimientos a escala y de la forma más cómoda para el cirujano. Magnifica los beneficios clínicos de la cirugía de mínima invasión. El tiempo de hospitalización es más corto, existe menos riesgo de infección, hay menor pérdida de sangre, menos transfusiones, cicatrices más pequeñas, menos dolor y una recuperación más rápida. 
Hoy dependemos de un gran número de tecnologías que, sin duda alguna, representan beneficios para el paciente y los médicos. Sin embargo, y con toda la convicción de que los cambios deben ser a favor de las nuevas tecnologías que demuestren ser beneficiosas, no debemos olvidar que detrás de estas máquinas se encuentran personas: el médico y el paciente. Si bien con estas nuevas tecnologías interactuamos con máquinas, no debemos olvidar lo importante que es la relación médico-paciente, base de todos los conocimientos actuales en medicina.

\section{REFERENCIAS}

[1] Krut, S. (2006). Introduction to Medical Robotics. Conferencia. VI Seminario de Automática. Popayán, Colombia.

[2] Salinas, S. (2009). "Modelado, simulación y control del robot para cirugía laparoscópica 'Lapbot”. Revista Chilena de Ingeniería, v. 17, n. 3, 317-328.

[3] Patiño, J., Cervantes, J. (1997). Cirugía Laparoscópica y Toracoscópica. México: McGrawHill Interamericana.

[4] Villavicencio, H. (2006). Cirugía laparoscópica avanzada robótica Da Vinci: origen, aplicación clínica actual en Urología y su comparación con la cirugía abierta y laparoscópica. Actas Urológicas Españolas, España.

[5] Davies, B. (2002). A review of robotics in surgery. EE. UU: Procinstmech Eng.

[6] Borden, L. Kozlowski, P. Porter, C. (2007). Mechanical failure rate of da Vinci robotic system. EE. UU.: Medline. 